Volume 111, Number 6, November-December 2006

Journal of Research of the National Institute of Standards and Technology

[J. Res. Natl. Inst. Stand. Technol. 111, 461 (2006)]

\title{
Errata
}

\section{Erratum: Noise-Parameter Uncertainties: A Monte Carlo Simulation}

\section{J. Randa}

National Institute of Standards and Technology, Boulder, CO 80305

james.randa@boulder.nist.gov

[J. Res. Natl. Inst. Stand. Technol., Volume 107, Number 5, September-October 2002, p. 433]

Erratum: In Eq. (6), the $X_{12}$ in the final term should instead be $X_{12}{ }^{*}$. Thus, the correct form of Eq. (6) is

$$
T_{1}=\frac{1}{\left(1-\left|\Gamma_{G S}^{\prime}\right|^{2}\right)}\left\{\frac{\left|S_{12}\right|^{2}\left(1-\left|\Gamma_{G}\right|^{2}\right)}{\left|1-\Gamma_{G} S_{22}\right|^{2}} T_{G}+\left|\frac{S_{12} S_{21} \Gamma_{G}}{1-\Gamma_{G} S_{22}}\right|^{2} X_{2}+X_{1}+2 \operatorname{Re}\left[\frac{S_{12} S_{21} \Gamma_{G} X_{12}^{*}}{1-\Gamma_{G} S_{22}}\right]\right\}
$$

Results in the paper are unchanged. I am grateful to Tom McKay of RF Micro Devices and Larry Wagner of IBM for calling this to my attention. 